\title{
Properties of Solar Wind Turbulence from Radio Occultation Experiments with the NOZOMI Spacecraft
}

\author{
A. I. Efimov' ${ }^{1}$ T. Imamura ${ }^{2}$ K.-I. Oyama ${ }^{3}$, K. Noguchi ${ }^{4}$, \\ L. N. Samoznaev ${ }^{1}$, A. S. Nabatov ${ }^{5}$, M. K. Bird ${ }^{6}$, and I. V. Chashei ${ }^{7}$ \\ ${ }^{1}$ Kotel'nikov Institute of Radio Engineering and Electronics, Russian Academy of Sciences, Moscow, Russia \\ ${ }^{2}$ Institute of Space and Astronautical Science, Japan Aerospace Exploration Agency, Sagamihara, Japan \\ ${ }^{3}$ Plasma and Space Science Center, National Cheng-Kung University, Tainan, Taiwan \\ ${ }^{4}$ Department of Information and Computer Science, Nara Women's University, Nara, Japan \\ ${ }^{5}$ Institute of Radio Astronomy, National Academy of Sciences of Ukraine, Khar'kov, Ukraine \\ ${ }^{6}$ Argelander Institute of Astronomy, University of Bonn, Bonn, Germany \\ ${ }^{7}$ Pushchino Radio Astronomy Observatory, Astro Space Center, Lebedev Physical Insitute, \\ Russian Academy of Sciences, Moscow, Russia \\ Received March 31, 2010; in final form, June 8, 2010
}

\begin{abstract}
Radio-sounding experiments using signals from the Japanese NOZOMI spacecraft to probe the circum solar plasma were performed from December 2000 through January 2001. They can be used to obtain information about the properties of the solar wind plasma in the region where it is accelerated at heliocentric distances of $12.8-36.9 R_{s}$ (where $R_{s}$ is the radius of the Sun). Measurements of the intensity and frequency of the received signals were carried out with high time resolution $(\sim 0.05 \mathrm{~s}$ for the frequency and $\sim 0.0064 \mathrm{~s}$ for the intensity), making it possible to investigate the anisotropy of inhomogeneities and the spatial spectrum of the turbulence of the circum solar plasma. Analysis of these radio-sounding data has shown that the scintillation index and intensity of the frequency fluctuations decrease approximately according to a power law with increasing distance of the line of sight from the Sun. Measurements of the amplitude fluctuations and estimates of the solar wind velocity derived from spatially separated observations indicate the presence of small-scale inhomogeneities with sizes of the order of $50 \mathrm{~km}$ at heliocentric distances less than $25 R_{s}$, which are elongated in the radial direction with anisotropy coefficients from 2.3 to 3.0. The inhomogeneities at heliocentric distances exceeding $30 R_{s}$ become close to isotropic.
\end{abstract}

DOI: $10.1134 / \mathrm{S} 1063772910110089$

\section{INTRODUCTION}

Much information about the parameters of the solar wind in its acceleration region, which is not accessible to direct measurements, can be inferred from the analysis of radio signals emitted by spacecraft and natural radio sources that pass through the solar wind plasma. Radio astronomy data and data from radio physics experiments have been used to find the characteristics of inhomogeneities (turbulence) of the solar wind plasma, which give rise to fluctuations of the amplitude, phase, and frequency of such radio waves. Radio sounding of the circum solar plasma has established that the index of the spatial spectrum of the plasma inhomogeneities $p$ depends on the heliocentric distance. In the region where the flow has formed (heliocentric distances exceeding $20 R_{s}$, where $R_{s}$ is the radius of the Sun), the index $p$ for the largescale component $\left(10^{3}-10^{6} \mathrm{~km}\right.$ in size)varies near 3.67 , close to a Kolmogorov spectrum. At heliocentric distances $R<15 R_{s}$, this index decreases to 3.0$3.2[1,2]$. According to certain as yet unconfirmed data, the small-scale portion of the turbulence spectrum may be flatter $[3,4]$. Analysis of radio-sounding data has also shown that the electron density inhomogeneities are anisotropic, and are elongated in the radial direction. The degree of anisotropy (the axial ratio of the spatial scales of the plasma density fluctuations) depends on heliocentric distance, the phase of the solar activity cycle, and the size of the inhomogeneities [5, 6].

Additional possibilities for investigating the smallscale structure of the solar wind are provided by hightime-resolution amplitude and phase measurements probing the circum solar plasma carried out with the Japanese NOZOMI spacecraft [7], as well as the simultaneous reception of such signals at two observing points and the determination of the solar wind velocity [8]. 
The current paper further develops the results of [7]. Our goal is to determine the characteristics of the turbulence of the solar wind plasma (the spatial spectrum and level of the turbulence and the degree of anisotropy), and to obtain the radial dependences of the scintillation index and intensity of the frequency fluctuations based on radio sounding of the solar wind using signals from the NOZOMI spacecraft.

\section{CHARACTERISTICS OF THE EXPERIMENTS}

Radio sounding of the circum solar plasma was carried out using NOZOMI signals from December 6, 2000 through January 23, 2001 [7]. The observations (CR 1970-1972) took place during maximum solar activity: the Wolf number exceeded 100 , and sunspots encompassed heliolatitudes $\pm 40^{\circ}$ (http://sidc.oma.be/html). The conditions on the Sun were fairly quiescent: only a few comparatively weak (class $M$ ) flares occurred during this period. Therefore, we infer that these NOZOMI experiments probed the region of circum solar plasma corresponding to the streamer belt. The sounding was carried out as the line of sight both approached the Sun (east limb) and receded from the Sun (west limb). Seven sessions were conducted from December 6 , to December 28, 2000, as the distance $R$ between the line of sight and the Sun decreased from $36.9 R_{s}$ to $12.8 R_{s}$. The distances $R$ in two sessions on January 22 and 23, 2001 as the line of sight receded from the Sun were $15.7 R_{s}$ and $16.9 R_{s}$. The probed regions were located near the plane of the solar equator.

The NOZOMI experiments were carried out in a coherent two-way mode. An S-band (wavelength $\lambda=13.1 \mathrm{~cm}$, carrier frequency $f=2.1123 \mathrm{GHz}$ ) signal radiated by ground-based systems passed near the Sun and was then received by systems on the spacecraft. On board the spacecraft, the frequency was multiplied by the coefficient $q=880 / 221$ to form an $\mathrm{X}$-band $(\lambda=3.6 \mathrm{~cm}, f=8.4109 \mathrm{GHz})$ response signal that was coherent with the original $\mathrm{S}$-band signal; this signal was then transmitted toward an observing point on Earth, where it was received. Fluctuations in the received $\mathrm{X}$-band signal are due to two factors: fluctuations in the S-band signal arising in its path from the original ground-based radiation point to the spacecraft, multiplied by the coefficient $q$, and fluctuations in the X-band signal arising during its propagation from the spacecraft to the ground-based reception point. Since the intensity of the frequency fluctuations due to the presence of plasma is inversely proportional to the frequency, the latter of these components is a factor of $q^{2}$ smaller than the former in power, and can be neglected. The fluctuations in the intensity (or amplitude) are due only to the influence of the plasma on the $\mathrm{X}$-band signal propagating from the spacecraft to the reception point.

An important property of the NOZOMI data is their high time resolution, which makes it possible to carry out studies of the spatial spectrum of the turbulence of small-scale inhomogeneities in the solar wind. The period of the frequency measurement cycle is $T=0.0512 \mathrm{~s}$ (corresponding to a sampling rate of $F=19.531 \mathrm{~Hz}$ ), while the period of the intensity measurement cycle was a factor of eight shorter $(T=$ $0.0064 \mathrm{~s}, F=156.25 \mathrm{~Hz}$ ). The time resolution used in the reduction of the NOZOMI data is much higher than in analogous experiments with the ULYSSES (1991, 1995) and GALILEO (1995-2004) spacecraft, where the period $T$ was $1 \mathrm{~s}$ [2].

\section{SPECTRAL ANALYSIS \\ OF THE FREQUENCY FLUCTUATIONS}

The spectral density of the fluctuation frequency $G_{f}(\nu)$ is related to the characteristics of the sounded turbulent plasma by the known expression [2]

$$
G_{f}(\nu) \propto \nu^{2} \exp \left(-\frac{\nu^{2}}{\nu_{m}^{2}}\right)\left(\nu_{0}^{2}+\nu^{2}\right)^{-\left(2+\alpha_{f}\right) / 2},
$$

where $\nu$ is the fluctuation frequency, $\nu_{0}=V / L_{0}$, $\nu_{m}=V / L_{m}$ are the fluctuation frequencies corresponding to the outer $L_{0}$ and inner $L_{m}$ turbulence scales, $V$ is the velocity of the solar wind, and $\alpha_{f}$ is the index of the fluctuation frequency spectrum, which is the power-law index of the three-dimensional spatial spectrum of the turbulence $p$ minus three [2].

Using known data on $L_{m}$ and $L_{0}$ and assuming $V=300 \mathrm{~km} / \mathrm{s}$, we can estimate the expected values for the frequencies $\nu_{0}$ and $\nu_{m}$. As follows from radio astronomy data [3] and radio physics experiments [9], the inner scale, on average, increases with heliocentric distance $R$ from $\sim 10 \mathrm{~km}$ at $R=10 R_{s}$ to $40 \mathrm{~km}$ at $R=30 R_{s}$; the frequency $\nu_{m}$ decreases accordingly, from $\sim 30 \mathrm{~Hz}\left(R=10 R_{s}\right)$ to $\sim 7.5 \mathrm{~Hz}\left(R=30 R_{s}\right)$. The outer scale also increases in a regular fashion with distance [10], from $\sim 2 R_{s}\left(R=10 R_{s}\right)$ to $\sim 6 R_{s}$ $\left(R=30 R_{s}\right)$, while the frequency $\nu_{0}$ varies from $\sim 2 \times$ $10^{-4}$ to $7 \times 10^{-5} \mathrm{~Hz}$. The NOZOMI experiments have the minimum possible value $\nu \gg \nu_{0}$; as follows from (1), the spectral density can be represented in the form

$$
G_{f}(\nu) \propto \nu^{-(p-3)} \exp \left(\frac{-\nu^{2}}{\nu_{m}^{2}}\right) .
$$

Figure 1 shows four temporal spectra of the frequency fluctuations for the signals received at the ground-based reception points on December 28, $2000\left(R=12.8 R_{s}\right)$, January $23,2001\left(R=16.8 R_{s}\right)$, December $18,2000\left(R=23.8 R_{s}\right)$, and December 11 , 


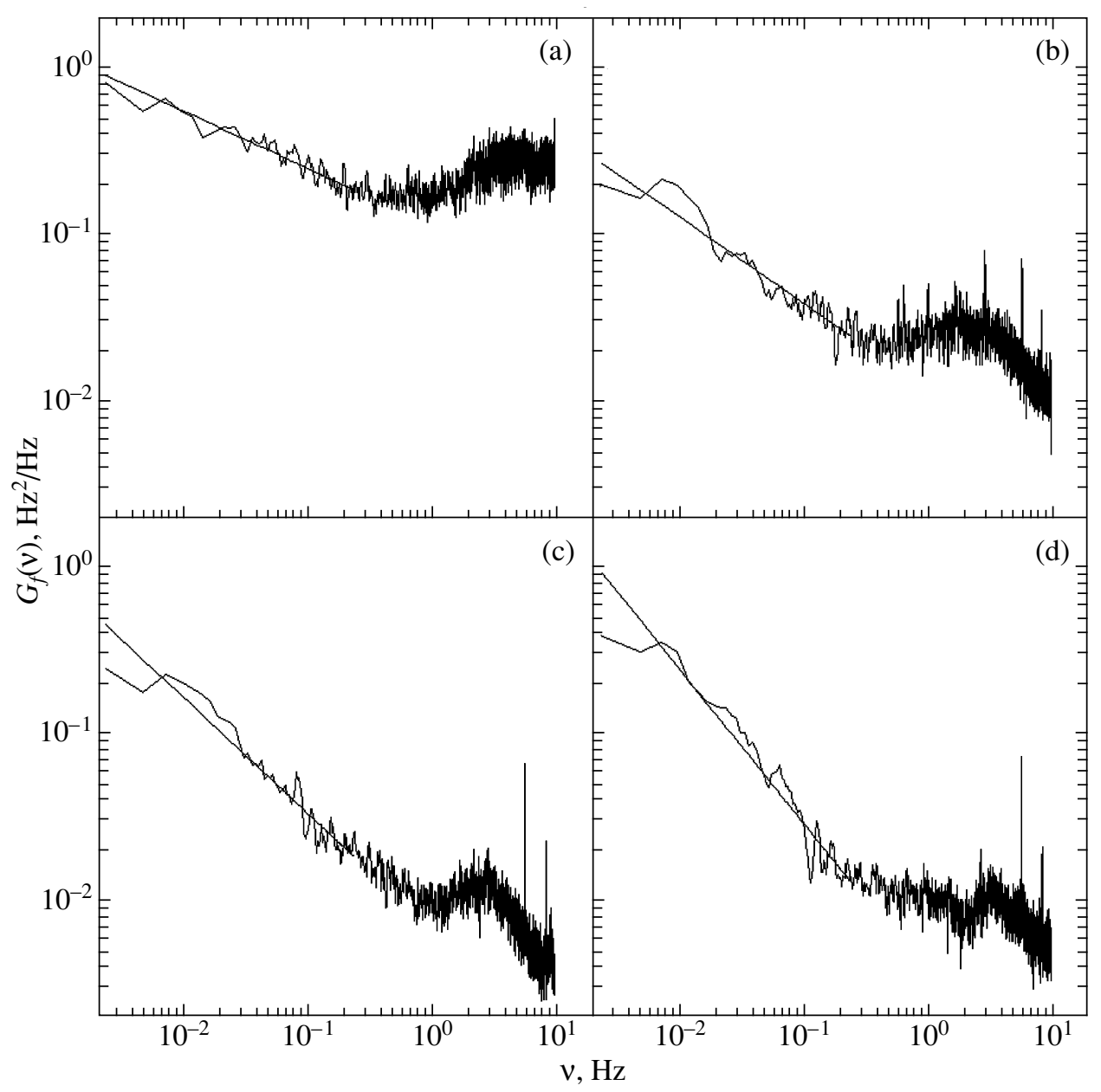

Fig. 1. Temporal spectra for the frequency fluctuations for (a) December $28,2000\left(R=12.8 R_{s}, \alpha_{f}=0.34\right)$, (b) January 23 , $2001\left(R=16.8 R_{s}, \alpha_{f}=0.56\right)$, (c) December $18,2000\left(R=23.8 R_{s}, \alpha_{f}=0.69\right)$, and (d) December $11,2000\left(R=31.5 R_{s}\right.$, $\left.\alpha_{f}=0.93\right)$.

$2000\left(R=31.5 R_{s}\right)$. The presented spectra characterize the frequency fluctuations for the S-band signal in the path from the ground-based radiation point to the spacecraft multiplied by the coefficient $q$. These were obtained by averaging an ensemble of individual spectra derived by applying a Fast Fourier Transform (FFT) in successive time segments with durations of 8192 counts covering the entire measurement session. The data of Fig. 1 show that the spectral density $G_{f}(\nu)$ for fluctuation frequencies $0.002 \mathrm{~Hz} \leq \nu \leq 0.5 \mathrm{~Hz}$ can be approximated by a power law. The approximating exponential relations are shown in Fig. 1 as linear functions on a loglog scale. The power-law index (spectral index) for these functions $\alpha_{f}$ varies over an appreciable range, from 0.34 (Fig. 1a) to 0.93 (Fig. 1d). In most cases, $\alpha_{f}$ varies around 0.67 , corresponding to Kolmogorov turbulence. Higher values of $\alpha_{f}$ could be associated with the passage of coronal mass ejections through the propagation path. These results agree with data obtained using other spacecraft $[1,2]$. This is true for the growth in $\alpha_{f}$ when perturbed flows of plasma pass through the propagation path (December 11, 2000; Fig. 1d) and the decrease (on average) of the spectral index of the frequency fluctuations that occurs when the line of sight of the radio signal approaches the Sun (December 28, 2000; Fig. 1a).

An important property of the spectra depicted in Fig. 1 is the excess spectral density of the frequency fluctuations in the interval $\nu=0.5-3 \mathrm{~Hz}$, compared to a power law. The detection of this effect was possible thanks to the high time resolution of the frequency measurements. This observed enhancement in the spectral density may be due to the influence of amplitude fluctuations. At fairly low fluctuation frequencies, where amplitude fluctuations are insignificant, the spectral density of the frequency $G_{f}(\nu)$ and phase 
$G_{\varphi}(\nu)$ fluctuations are related as [2]

$$
G_{\varphi}(\nu)=\frac{G_{f}(\nu)}{\nu^{2}}
$$

making it easy to find the phase spectra from the frequency spectra. However, this relation must be modified at frequencies comparable to the Fresnel frequency, since, in the standard definition of the phase and frequency,

$$
\begin{gathered}
\varphi_{1}(t)=-i \ln \left(\frac{A(t)}{A_{0} \exp (i \varphi(t))}\right), \\
2 \pi f_{1}(t)=\frac{d \varphi_{1}}{d t}-2 \pi f_{0},
\end{gathered}
$$

where $\varphi_{1}(t)$ and $f_{1}(t)$ are the modified phase and frequency, respectively, $A(t)$ and $A_{0}$ are the instantaneous amplitude of the signal and its mean unperturbed value, and $f_{0}$ is the carrier frequency of the sounding radio signal. Relation (4) can be used to determine the correlation function of the fluctuations,

$$
\begin{gathered}
B_{f}(\tau)=\operatorname{Re}\left\langle f_{1}(t) f_{1}^{*}(t+\tau)\right\rangle \\
=\frac{d^{2}}{d t^{2}}\left(B_{\varphi}(\tau)+B_{\chi}(\tau)\right),
\end{gathered}
$$

where $B_{\varphi}(\tau)$ and $B_{\chi}(\tau)$ are the correlation functions of the phase $\varphi(t)$ and the level $\chi(t)=\ln \left[A(t) / A_{0}\right]$. In contrast to (3), the temporal spectrum of the fluctuation frequency corresponding to (5) is

$$
G_{f}(\nu)=\nu^{2}\left[G_{\varphi}(\nu)+G_{\chi}(\nu)\right],
$$

and depends on both the spectra $G_{\varphi}(\nu)$ and $G_{\chi}(\nu)$. The form of the frequency-fluctuation spectra in Fig. 1 exactly corresponds to (6): at low frequencies, where the signal-level fluctuations are low compared to the phase fluctuations, the spectra are close to power-law. The difference of the spectra from power laws at $0.5-3 \mathrm{~Hz}$ is associated with the influence of the signal-level fluctuations, whose spectra (see Fig. 5) are close to flat at frequencies below the Fresnel frequency for the $\mathrm{S}$ band $(\sim 0.5 \mathrm{~Hz})$. The possibility of a contribution of amplitude fluctuations to the temporal spectra of the modified frequency is also supported by estimates of the scintillation index in the S band. When the X-band scintillation indices presented in Fig. 6 below are recalculated to the $\mathrm{S}$ band taking into account the expected wavelength dependence, $\sigma_{i} \propto \lambda^{(p+2) / 4}$ [11], these correspond to values $1.0 \geq \sigma_{i} \geq 0.36$ at heliocentric distances $(13-30) R_{S}$; i.e., the amplitude fluctuations in the $\mathrm{S}$ band are substantial. At the same time, measurements of the intrinsic frequency fluctuations with averaging times shorter than the characteristic time scale for the fluctuation amplitude become difficult, especially in the case of saturated amplitude fluctuation, when the mean wave field becomes close to zero and the scintillation index equal to unity.

Figure 2 shows four modified phase fluctuation spectra derived for the same sessions, on December 28, 2000, January 23, 2001, December 18, 2000, and December 11, 2000. At frequencies below $0.1 \mathrm{~Hz}$, the modified phase fluctuation spectra are close to power laws and close to the phase fluctuation spectra. At frequencies above $0.5 \mathrm{~Hz}$, the spectra are broadened by the amplitude fluctuations. An interesting feature of the spectra presented on the log-log scale in Fig. 2 is that they become nearly parallel at frequencies below $0.1 \mathrm{~Hz}$ and above $3 \mathrm{~Hz}$. This property is due to the fact that the power-law indices for the amplitude and phase fluctuation spectra are equal at frequencies above the Fresnel frequency. Figure 3 shows the values of $p$ determined from the frequency fluctuations at $0.002 \mathrm{~Hz} \leq \nu \leq 0.1 \mathrm{~Hz}$ as a function of $R$. On average, the power-law index $p$ is close to 3.67 , characteristic of Kolmogorov turbulence, with no obvious dependence on the heliocentric distance. Only the value of $p$ in the innermost point of the studied range of distances from the Sun $\left(\sim 13 R_{S}\right)$ is slightly lower, $p=3.38$.

The dependence of the rms frequency fluctuations on the distance of the line of sight from the Sun $R$ was obtained from our spectral analysis of the data. Figure 4 presents the values of $\sigma_{f}(R)$ for the S-band signal on a log-log scale, obtained by integrating the spectral density from the minimum frequency $\nu$ to $\nu=3.0 \mathrm{~Hz}$. The $\sigma_{f}(R)$ can be approximated using a power law:

$$
\sigma_{f}(R)[\mathrm{Hz}]=B\left(R / R_{s}\right)^{-b},
$$

where $B=35.80$ and $b=1.61$. This power-law function is shown by the straight line in Fig. 4. This value of $b$ is in agreement with data obtained using other spacecraft, which indicate power-law indices $b$ in the broad range 1.5-2.5 [12].

\section{SPECTRAL ANALYSIS OF THE AMPLITUDE FLUCTUATIONS}

The signal level is determined by the ratio of the amplitudes of the radio waves propagating in the inhomogeneous medium $\mathrm{A}$ and in the unperturbed medium $A_{0}$ :

$$
\chi=\ln \left(\frac{A}{A_{0}}\right) .
$$

For small fluctuations, the spectral density of the signal-level fluctuation $G_{\chi}(\nu)$ can be found from the temporal spectrum of the intensity fluctuations $G_{i}(\nu)$ using the simple relation

$$
G_{\chi}(\nu)=0.25 G_{i}(\nu)
$$




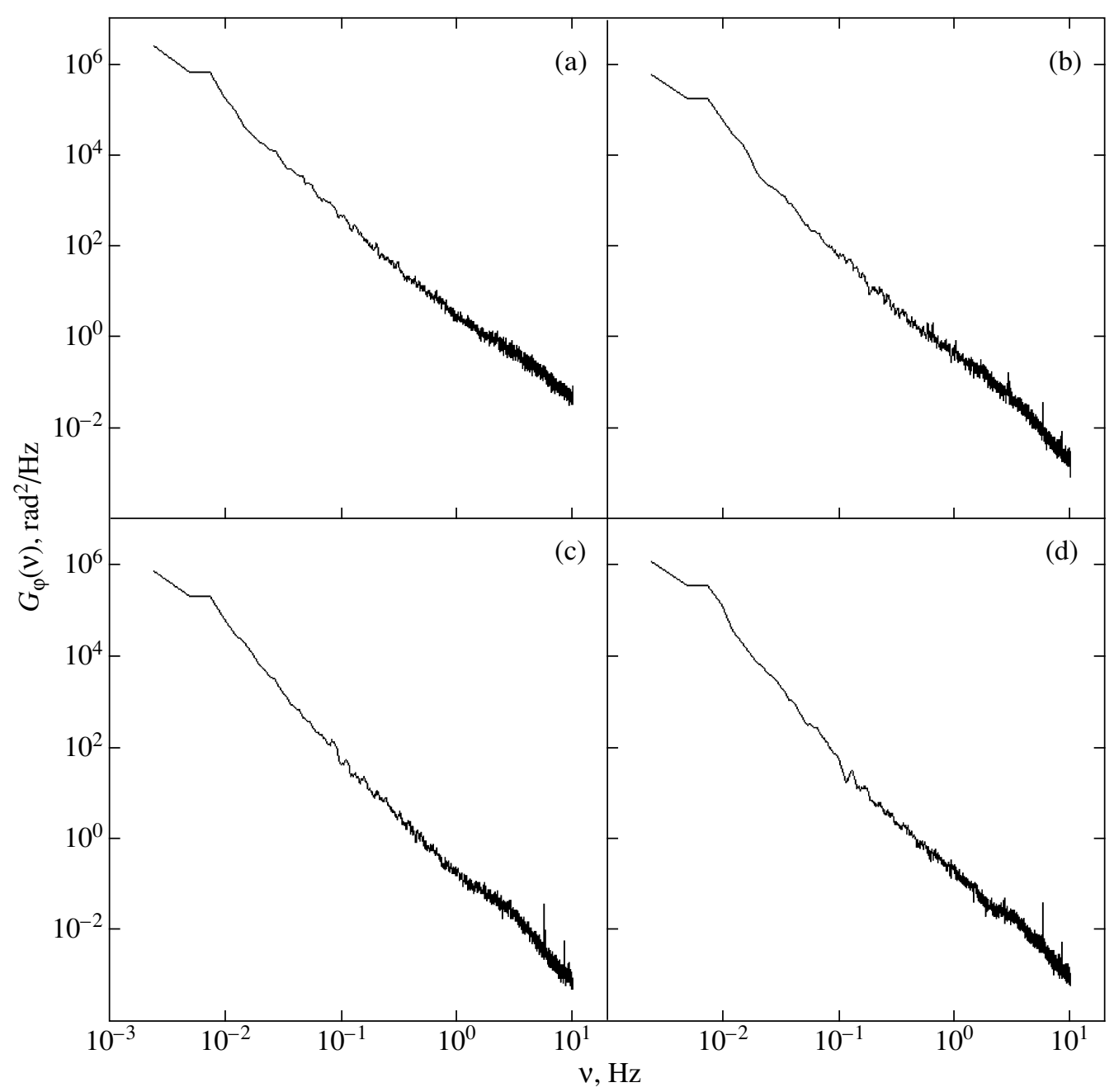

Fig. 2. Temporal spectra of the phase fluctuations for (a) December 28, 2000 ( $\alpha_{\varphi}=2.38$ ), (b) January 23, 2001 ( $\alpha_{\varphi}=2.64$ ), (c) December 18, 2000 ( $\left.\alpha_{\varphi}=2.73\right)$, (d) December 11, $2000\left(\alpha_{\varphi}=2.95\right)$.

Figure 5 shows four temporal spectra for the intensity fluctuations of the X-band signal calculated from the radio-sounding measurements on December $28,2000\left(R=12.8 R_{s}\right)$, December $23,2000(R=$ $\left.18.3 R_{s}\right)$, December $18,2000\left(R=23.8 R_{s}\right)$, and December $6,2000\left(R=36.9 R_{s}\right)$. The spectra were obtained by averaging individual spectra in successive sets of 8192 counts covering the entire session. The data presented in Fig. 5 show that the scintillation is enhanced as the line of sight approaches the Sun, and the spectral density $G_{i}$ increases. The spectra are informative up to some frequency $\nu_{1}$, above which they become noisy. Depending on $R$, the frequency $\nu_{1}$ varies in the range $5-15 \mathrm{~Hz}$.

Typical spectra of the scintillation amplitudes have a flat section at low frequencies and fall off approximately according to a power law at high frequencies. The location of the intersection of the asymptotes approximating the low-frequency and high-frequency temporal spectra for the amplitudes and intensities yields the characteristic frequency $\nu_{c}$, given by $[13$, 14]

$$
\nu_{c}=\frac{V_{a p p}}{\sigma_{p} r_{F}},
$$

where $\sigma_{p}$ is a weakly varying function of the index $p$ (it increases from $\sim 2.2$ to 2.6 [6] as $p$ varies from 3.0 to 4.0 ),

$$
r_{F}=\sqrt{\lambda \frac{L_{1} L_{2}}{L_{1}+L_{2}}}
$$

is the radius of the first Fresnel zone, $L_{1}$ is the distance between the scattering layer containing the inhomogeneities and the spacecraft, $L_{2}$ is the distance between the scattering layer and the groundbased station, and $V_{a p p}$ is the apparent velocity of the inhomogeneities through the line of sight (i.e., the apparent velocity of the diffraction pattern). If 


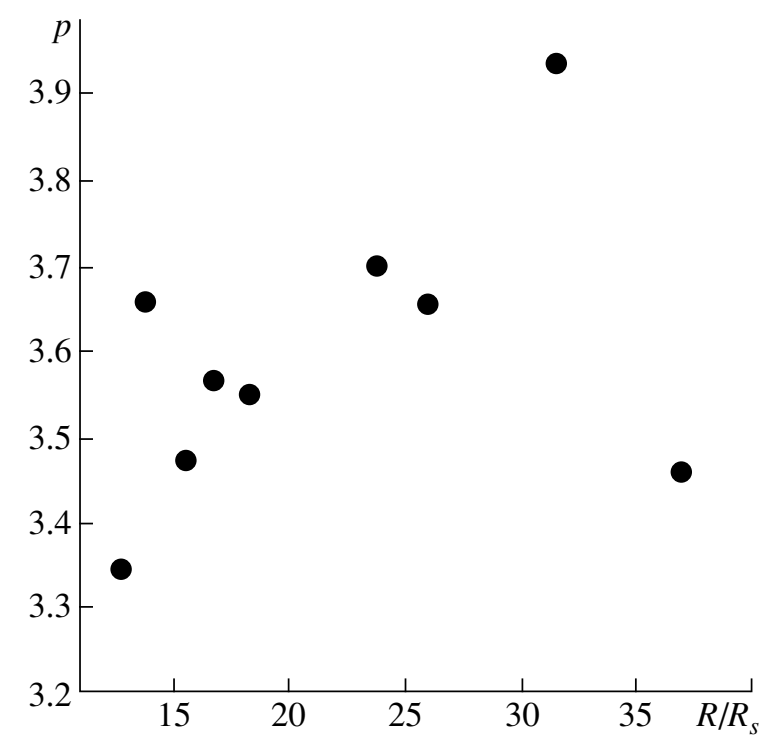

Fig. 3. Index for the three-dimensional spatial spectrum of the turbulence $p$ as a function of heliocentric distance $R$.

the anisotropy parameter $\zeta=1$ and the solar wind velocity $V$ is much higher than the sound speed, as is true at fairly large distances from the Sun, then $V_{a p p}=V$ [6]. In this case, relation (10) indicates the possibility of determining the solar wind velocity from the characteristic frequency $\nu_{c}$ [14]. The ratio $V / V_{\text {app }}$ represents an upper limit for $\zeta$, namely $\zeta \leq$ $V / V_{\text {app }}[6]$.

Figure 6 presents the rms values of the intensity fluctuations (or scintillation index) of the X-band signal $\sigma_{i}$ as a function of the heliocentric distance $R$. The $\sigma_{i}$ values were obtained by integrating the spectral density $G_{i}$ from the lowest frequency $(\nu \approx 0.02 \mathrm{~Hz})$ to $\nu_{1}$. The dependence $\sigma_{i}(R)$ (like $\sigma_{f}(R)$ ) can also be approximated using a power law of the form (7), with $B=4.64$ and $b=1.305$; this function is shown by the straight line on the log-log plot in Fig. 6.

During the propagation of a plane radio wave in a turbulent medium, the signal-level and phase spectra are identical at high frequencies $\left(\nu>\nu_{c}\right)$ [15]. Simultaneous measurements of the amplitude and frequency of the sounding signal with a small time step can be used to verify this theoretical expectation. Figure 7 shows the spectra of the phase fluctuations $G_{\varphi}$ (curve 1 ) and the signal-level fluctuations $G_{\chi}$ (curve 2) for X-band measurements carried out on January 23, 2001. The spectral density of the phase fluctuations was determined from the spectrum of the received signal by dividing it by $q^{2}$. Both spectra are the result of averaging an ensemble of individual spectra obtained in a succession of time segments

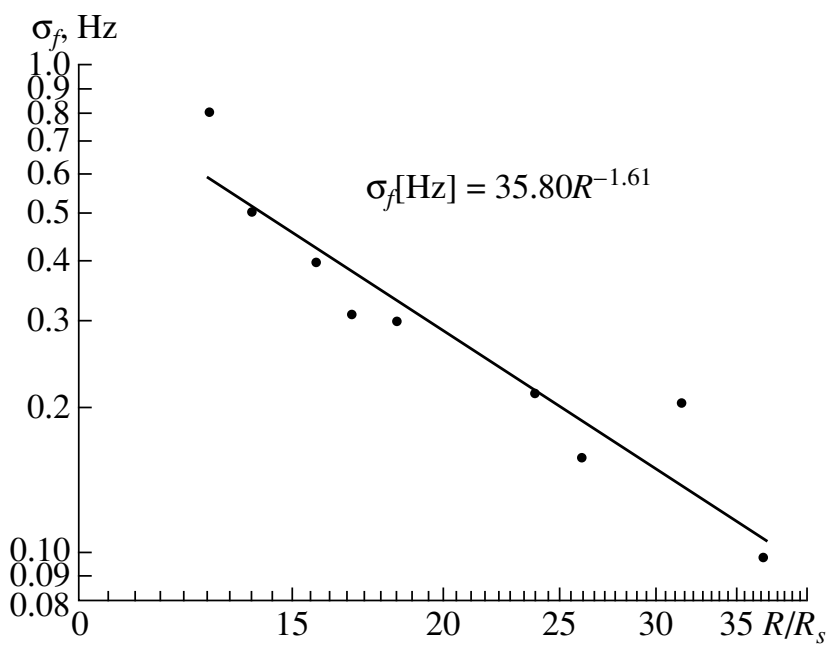

Fig. 4. Root-mean-square values $\sigma_{f}$ (points) of the frequency fluctuations for the S-band signal for various heliocentric distances $R$. The straight line shows the result of a least-squares fit.

during the entire measurement session. The FFT1024 and FFT-128 algorithms were used to find the spectral density of the signal-level fluctuations and phase fluctuations, respectively. To increase the accuracy with which the spectral density of the signallevel fluctuations is found from the derived spectra, we subtracted the mean noise level in the interval of fluctuation frequencies $\nu>\nu_{1}$.

A comparison of the curves in Fig. 7 shows that the behaviors of the phase and amplitude spectra are indeed similar, and their values are numerically close, although $G_{\varphi}>G_{\chi}$. A similar picture is observed for the other sessions. Only in one case (January 22, 2001 ) was the difference between $G_{\chi}$ and $G_{\varphi}$ substantial. This regularly observed difference in the experimental values of $G_{\chi}$ and $G_{\varphi}$ can be explained by the influence of the sphericity of the radio waves. As follows from relations derived in $[13,14]$, the sphericity of the waves reduces the dispersion of the fluctuations $\sigma_{\chi}^{2}$ by an amount $L_{1} /\left(L_{1}+L_{2}\right)^{(2-p) / 2}$ compared to the dispersion for a plane wave, which is a fairly strong dependence on $p$ : this quantity is 1.56 when $p=3.67$ and 1.18 when $p=2.6$. These values are comparable to the ratio $G_{\varphi} / G_{\chi}$. For example, when $\nu=3 \mathrm{~Hz}$, the ratio of the spectral densities (Fig. 7) is 1.34 .

The presence of so-called Fresnel minima and maxima located at fluctuation frequencies $\nu>\nu_{c}$ is characteristic of the amplitude spectra [14]. In particular, the first Fresnel minimum of the spectral density is at the frequency

$$
\nu_{\min }=\frac{V_{a p p}}{r_{F}},
$$




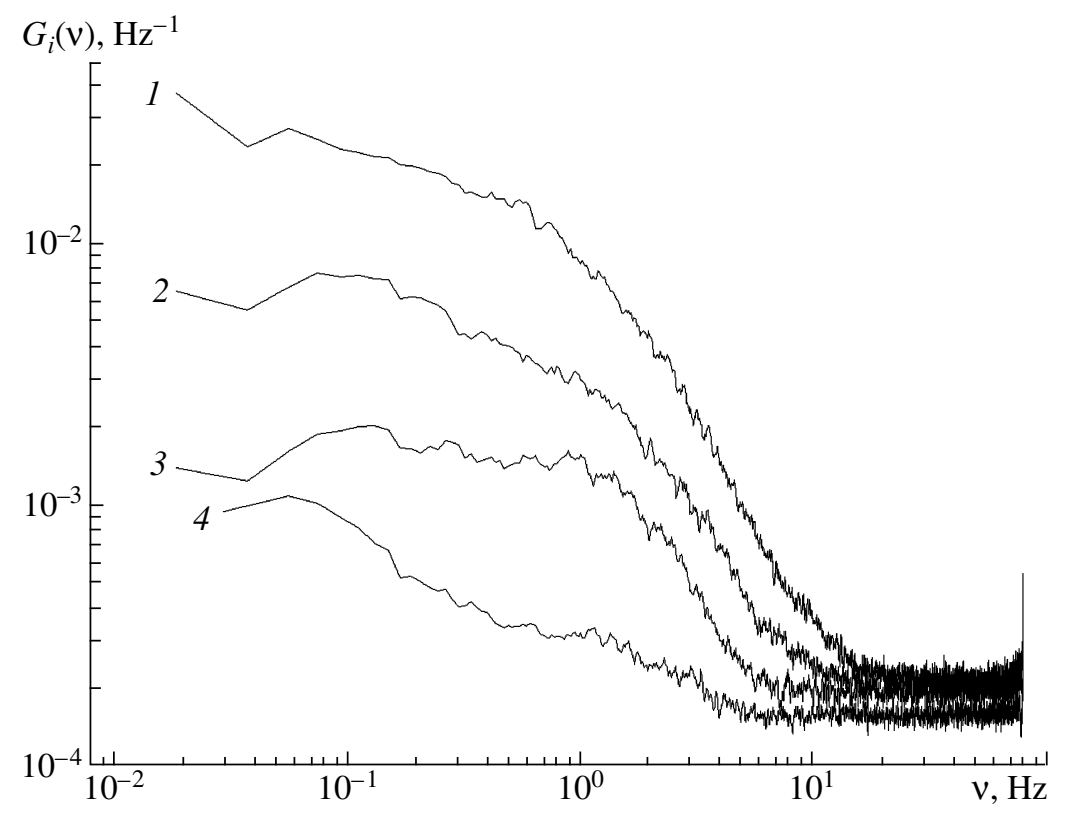

Fig. 5. Temporal spectra of the intensity fluctuations of the X-band signal for $(1)$ December $28,2000\left(R=12.8 R_{s}\right)$, (2) December 23, $2000\left(R=18.3 R_{s}\right)$, (3) December 18, $2000\left(R=23.8 R_{s}\right)$, and (4) December 6, $2000\left(R=36.9 R_{s}\right)$.

with $\nu_{\min } / \nu_{c}=\delta$. Thus, determining the value $\nu_{\min }$ from the experimental amplitude spectrum $G_{\chi}(\nu)$, we can find the velocity $V_{a p p}$, then use the ratio $V / V_{a p p}$ to derive an upper limit on the degree of elongation of the inhomogeneities in the radial direction.

The Fresnel minima and maxima are weakly expressed, and are not observed in spectra averaged over a large number of data records, as can be seen from the spectra presented in Figs. 5 and 7 . The Fresnel extrema can be made to appear by varying the number

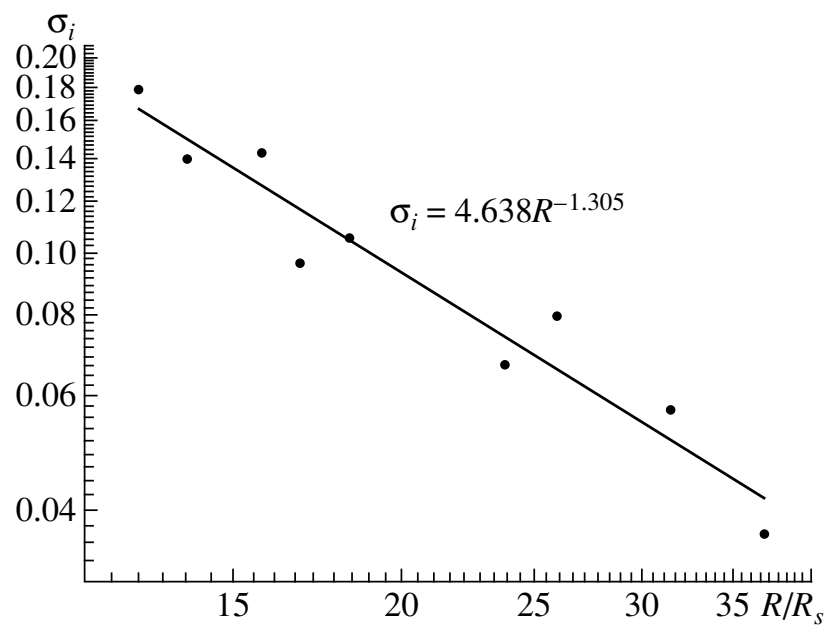

Fig. 6. Root-mean-square values $\sigma_{i}$ (points) of the intensity fluctuations for the X-band signal for various heliocentric distances $R$. The straight line shows the result of a least-squares fit. and durations of the data records. Figure 8 presents two amplitude spectra derived from measurements on December 28, 2000 (Fig. 8a) and December 23, 2000 (Fig. 8b), in which the first Fresnel minimum and maximum are visible. Both spectra were obtained using the FFT-2048 algorithm, via the averaging of eight data records. By means of illustration, curves approximating the low-frequency and high-frequency parts of the spectrum are shown in Fig. 8a. These

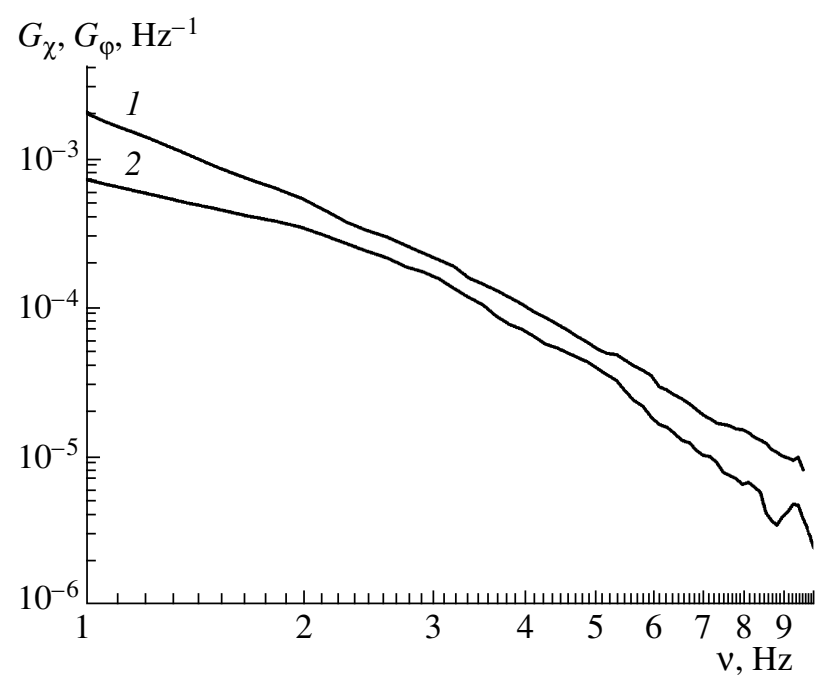

Fig. 7. Comparison of the temporal spectrum of the phase fluctuations (curve 1 ) and the spectrum of the signallevel fluctuations (curve 2) for X-band measurements on January 23, 2001. 


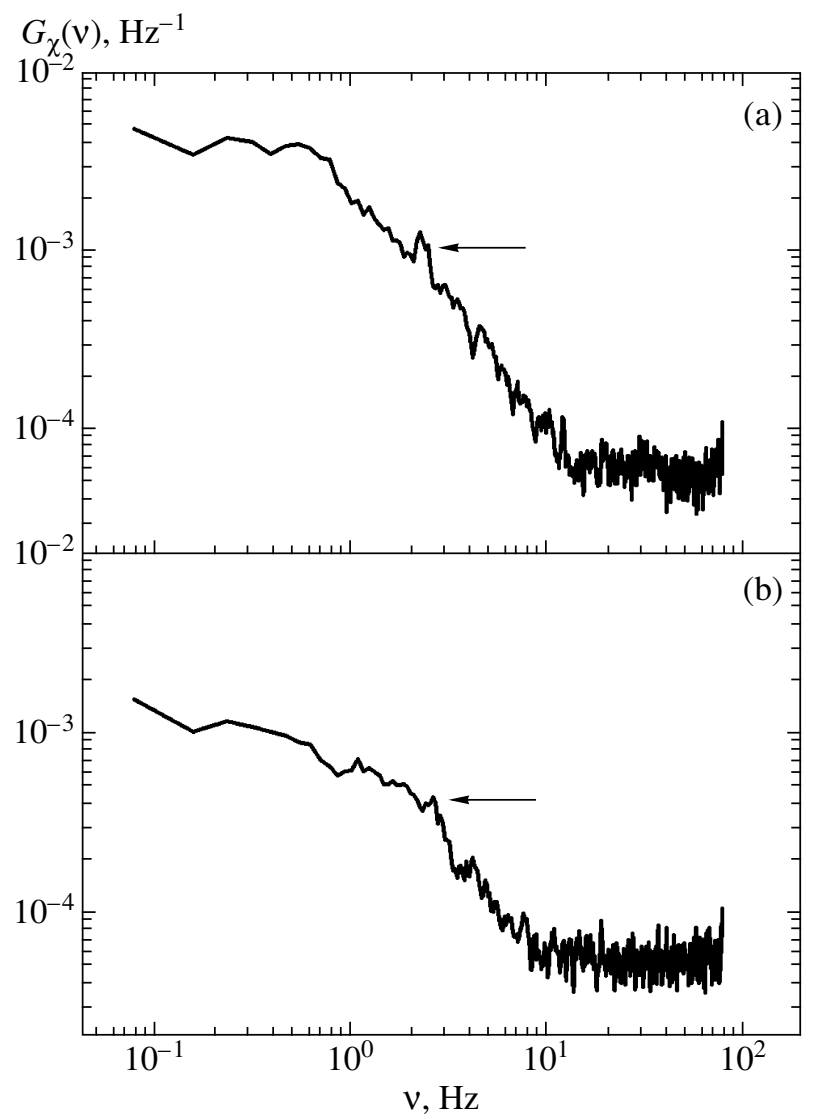

Fig. 8. Spectra of the signal-level fluctuations for (a) December $28,2000\left(\nu_{\min }=2.05 \mathrm{~Hz}\right)$ and (b) December 23,2000 $\left(\nu_{\min }=2.23 \mathrm{~Hz}\right)$.

intersect at $\nu=\nu_{c} \approx 0.84 \mathrm{~Hz}$. The Fresnel minimum is observed at $\nu_{\min } \approx 2.05 \mathrm{~Hz}$, so that $\nu_{\min } / \nu_{c} \approx 2.4$. Using the fact that $r_{F}=56 \mathrm{~km}$ together with formula (12), we can find the velocity of the diffraction pattern: $V_{a p p}=115 \mathrm{~km} / \mathrm{s}$.

To obtain an upper limit for the anisotropy parameter $\zeta$, we used data on the velocity of the solar wind obtained for six days in December 2000 (December $6,11,18,23,27$, and 28) via observations of the amplitude fluctuations made at two spatially separated points [8]. In particular, we find for December 28,2000 the velocity $V \approx 270 \mathrm{~km} / \mathrm{s}$, and accordingly $\zeta=2.35$. Values for $\zeta$ were found in the same way for December 18, $2000(\zeta=2.65)$, December $23,2000(\zeta=2.64)$, and December $27,2000(\zeta=$ $2.96)$. Thus, the anisotropy parameter $\zeta$ varies in the range $2.35 \leq \zeta \leq 2.96$ in the interval of heliocentric distances $(12.8-23.8) R_{s}$.

As the line of sight recedes from the Sun, the Fresnel extrema become less clearly expressed, and they are essentially indistinguishable in the spectra derived for December 6, 2000 and December 11, 2000, when $R>30 R_{s}$. In these two cases, the anisotropy parameter $\zeta$ is determined from the characteristic frequency $\nu_{c}$ using (10). The value of $\sigma_{p}$ was taken to be 2.2 (the theoretical value for $p=3$ ). Figure 9 presents spectra derived from measurements made on December 11 , 2000 (Fig. 9a) and December 6, 2000 (Fig. 9b). These spectra were obtained using the FFT-1024 algorithm by averaging ensembles of temporal spectra corresponding to a large number of data records (more than 500) during the entire session and subtracting the spectral density for the noise fluctuations. This method was adopted to obtain the maximum possible accuracy in $G_{\chi}$ and the frequency $\nu_{c}$. We used the spectra shown in Fig. 9 to find the values of $\nu_{c}$ and $V_{a p p}$. For the measurements of December 6, 2000, $\nu_{c}=2.8 \mathrm{~Hz}$ and $V_{a p p}=330 \mathrm{~km} / \mathrm{s}$, while $\nu_{c}=$ $3.05 \mathrm{~Hz}$ and $V_{a p p}=375 \mathrm{~km} / \mathrm{s}$ for the measurements of December 11, 2000. Both velocities $V_{a p p}$ are close to the corresponding solar wind velocities [8], indicating that the anisotropy parameter $\zeta$ is close to unity.

\section{CONCLUSION}

Our analyses of the temporal spectra for phase, amplitude, and frequency fluctuations of radiosounding signals detected in high-time-resolution 


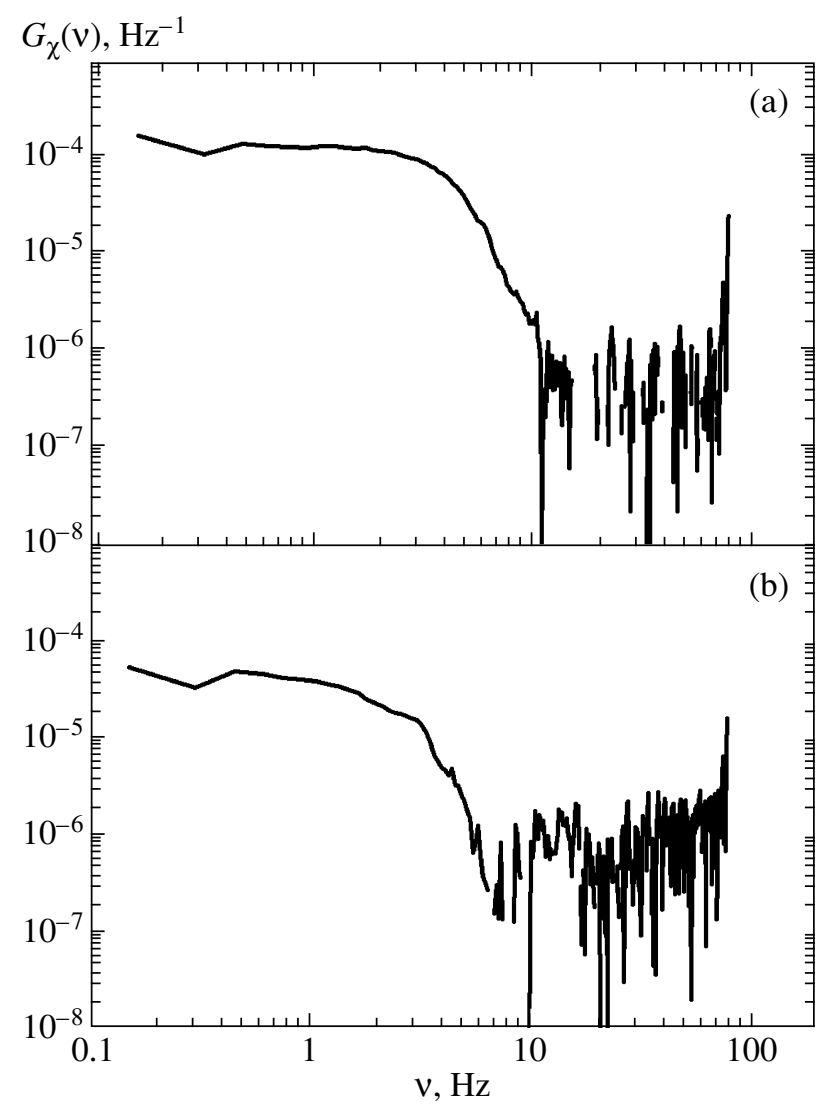

Fig. 9. Spectra of the signal-level fluctuations for (a) December 11, $2000\left(\nu_{\mathrm{c}}=3.05 \mathrm{~Hz}\right)$ and (b) December 6, 2000 $\left(\nu_{\mathrm{c}}=2.80 \mathrm{~Hz}\right)$.

measurements with the NOZOMI spacecraft have enabled us to study the turbulence of the small-scale component of the solar wind plasma.

We have increased the accuracy with which we determine the characteristics of the turbulence of the sounding plasma using a method based on averaging an ensemble of spectra corresponding to a large (about 500) number of data records. We subtracted the spectral density of the noise fluctuations from the total spectral density of the amplitude fluctuations for the sounding signals probing the solar wind.

We also tested a method for finding the degree of anisotropy of the plasma density inhomogeneities and their elongation in the radial direction. This method is based on the relationship between the solar wind velocity derived from observations of the signal-level fluctuations at two spatially separated observation points and the position on the frequency axis of the Fresnel minimum of the spectral density of the signal level.

The behavior of the temporal spectra of the frequency and phase fluctuations of the NOZOMI signals testifies that the index of the spatial spectrum of the turbulent solar wind plasma is close to Kolmogorov at heliocentric distances from $15 R_{s}$ to $30 R_{s}$, possibly becoming flatter closer to the Sun.

The degrees of elongation of small-scale plasma density inhomogeneities at heliocentric distances of $(13-25) R_{s}$ are $2.3-3$. The inhomogeneities are isotropic at distances exceeding $30 R_{s}$, in agreement with the results of radio astronomy observations [1618 .

We have obtained the radial dependences of the rms fluctuations of the frequency and the signal level at heliocentric distances $(13-37) R_{s}$. Both these dependences can be approximated by power laws with indices of -1.6 and -1.3 , respectively.

\section{ACKNOWLEDGMENTS}

This work was supported by a collaboration between the Russian Foundation for Basic Research and the Deutsche Forschungsgemeinschaft (DFG) (project 09-02-91337) the Russian Foundation for Basic Research (project 10-02-00078a), and the Basic Research Program of the Division of Physical Sciences of the Russian Academy of Sciences "Plasma Processes in the Solar System". The authors are 
thankful to M.A. Livshits for drawing their attention to the specific character of conditions on the Sun during the measurement period.

\section{REFERENCES}

1. R. Woo and J. W. Armstrong, J. Geophys. Res. 84, 7288 (1979).

2. N. A. Armand, A. I. Efimov, L. N. Samoznaev, et al., Radiotekh. i Elektron. 48, 1058 (2003). [J. Communic. Technol. Electr. 48, 970 (2003)].

3. W. A. Coles and J. K. Harmon, Astrophys. J. 337, 1023 (1989).

4. G. L. Tyler, J. F. Vesecky, M. A. Plume, H. T. Howard, and A. Barnes, Astrophys. J. 249, 318 (1981).

5. J. W. Armstrong, W. A. Coles, S. R. Spangler, et al., Astrophys. J. 358, 685 (1990).

6. A. I. Efimov, V. K. Rudash, M. K. Bird, et al., Adv. Space Res. 26, 785 (2000).

7. T. Imamura, K. Noguchi, A. Nabatov, et al., Astron. Astrophys. 439, 1165 (2005).

8. M. Tokumaru, K. Fujiki, H. Higashiyama, et al., unpublished manuscript (2004).

9. O. I. Yakovlev, A. I. Efimov, and S. N. Rubtsov, Astron. Zh. 65, 1290 (1988) [Sov. Astron. 32, 672 (1988)].
10. A. I. Efimov, I. V. Chashei, L. N. Samoznaev, et al., Astron. Zh. 79, 640 (2002) [Astron. Rep. 46, 579 (2002)].

11. A. I. Efimov, N. A. Armand, L. A. Lukanina, et al., Radiotekh. i Elektron. 53, 1257 (2008). [J. Communic. Technol. Electr. 53, 1186 (2008)].

12. A. I. Efimov, L. N. Samoznaev, M. K. Bird, et al., Adv. Space Res. 42, 117 (2008).

13. N. A. Armand, A. I. Efimov, and O. I. Yakovlev, Problems of Contemporary Radio Engineering and Electronics (Nauka, Moscow, 1987) [in Russian].

14. O. I. Yakovlev, Propagation of Radio Waves in Space (Nauka, Moscow, 1985) [in Russian].

15. R. Woo, Astrophys. J. 201, 238 (1975).

16. K. R. Grall, W. A. Coles, S. R. Spangler, et al., J. Geophys. Res. 102, 263 (1997).

17. Y. Yamauchi, M. Tokumaru, M. Kojima, et al., J. Geophys. Res. 103, 6571 (1998).

18. I. V. Chashei, A. I. Efimov, V. K. Rudash, and M. K. Bird, Astron. Zh. 77, 713 (2000) [Astron. Rep. 44, $634(2000)]$.

Translated by D. Gabuzda 